\title{
Zebrafish-A Model Organism for Studying the Neurobiological Mechanisms Underlying Cognitive Brain Aging and Use of Potential Interventions
}

\author{
Michelle M. Adams ${ }^{1,2,3,4,5 *}$ and Hulusi Kafaligonul ${ }^{1,5}$ \\ ${ }^{1}$ Interdisciplinary Neuroscience Program, Aysel Sabuncu Brain Research Center, Bilkent University, Ankara, Turkey, \\ ${ }^{2}$ Department of Psychology, Bilkent University, Ankara, Turkey, ${ }^{3}$ National Nanotechnology Research Center (UNAM), Bilkent \\ University, Ankara, Turkey, ${ }^{4}$ Department of Molecular Biology and Genetics Department Zebrafish Facility, Bilkent University, \\ Ankara, Turkey, ${ }^{5}$ National Magnetic Resonance Research Center (UMRAM), Aysel Sabuncu Brain Research Center, Bilkent \\ University, Ankara, Turkey
}

Keywords: aging, cognition and perception, behavior, neurobiological alterations, interventions, dietary restriction

\section{OPEN ACCESS}

Edited by:

Eirini Trompouki,

Max-Planck-Institut für Immunbiologie und Epigenetik, Germany

Reviewed by:

Claudia Vianna Maurer-Morelli, Universidade Estadual de Campinas,

Brazil

*Correspondence:

Michelle M. Adams

michelle@bilkent.edu.tr

Specialty section:

This article was submitted to

Molecular Medicine

a section of the journal

Frontiers in Cell and Developmental

Biology

Received: 09 August 2018 Accepted: 25 September 2018 Published: 01 November 2018

Citation:

Adams MM and Kafaligonul H (2018)

Zebrafish-A Model Organism for

Studying the Neurobiological Mechanisms Underlying Cognitive

Brain Aging and Use of Potential

Interventions.

Front. Cell Dev. Biol. 6:135.

doi: 10.3389/fcell.2018.00135
Classically, the zebrafish model organism has been used to elucidate the genetic and cellular mechanisms related to development since the embryo forms and grows externally following fertilization. This provides insight into the genetic control of developmental processes in humans because their genomes are similar. Also, unlike other animal models, the genes of zebrafish can be manipulated quite easily by using reverse genetic screens tools such as morpholinos, which transiently silence target genes of interest or systems such as the transposon-mediated insertional mutagenesis or CRISPR-Cas9. Moreover, one pair of fish will provide up to 300 offspring, which means that if there is a gene of interest that is manipulated, then it can be transmitted to a large population of fish. What is beginning to emerge is that similar to other mammals, adult zebrafish have an integrated nervous system, which is proposed to contain homologous brain structures to those found in humans, as well as equivalent cellular and synaptic structure and function. Moreover, like humans, zebrafish exhibit age-related declines in cognitive functions, and a convergence of evidence has indicated that subtle changes in cellular and synaptic integrity underlie these changes. Therefore, the zebrafish is a powerful model organism for studying the neurobiological consequences of aging-related behavioral and biological changes, which offers the potential to identify possible interventions that would promote healthy aging. In what follows, we present and discuss recent findings and advances along these directions.

\section{BEHAVIORAL TASKS AND ABILITIES ALTERED IN AGED ZEBRAFISH}

The zebrafish is a promising model for studying age-related changes in cognition and perception. Early behavioral studies date back to 1960s and the characterization of zebrafish behavior has accelerated since 2000 (Kalueff et al., 2013). They have been suggested to reflect the evolutionarily conserved nature of many behaviors and to resemble those of other species (Kalueff et al., 2014; Stewart et al., 2014; Orger and de Polavieja, 2017). A rich repertoire of behavioral phenotypes has been identified for cognitive functioning, perceptual processes, and associated disorders (Stewart and Kalueff, 2012). Using different behavioral assays (e.g., inter- and intratrial habituation, T-maze, conditioned place preference paradigms), previous studies indicated that zebrafish have both simple and relatively complex forms of learning, and also display good 
performance on cognitive tasks dependent on short-term and long-term memory (Blaser and Vira, 2014; Gerlai, 2016). There is also growing interest in other aspects of zebrafish behavior which significantly depend on perception, low-level discrimination, and sensitivity (Neuhauss, 2010). For instance, the basic components of the zebrafish visual system, the visual processing hierarchy, and pathways are similar to those commonly found in other species (Bilotta and Saszik, 2001). In particular, most of the previous research evaluated visual motion perception and sensitivity through optomotor response and/or optokinetic reflexive eye movements. These behavioral studies point to qualitatively similar visual acuity and contrast sensitivity functions for zebrafish (Rinner et al., 2005; Haug et al., 2010; Tappeiner et al., 2012). It has also been shown that zebrafish perceive first- and second-order motion. They also experience motion illusions commonly used in studies on human vision such as reverse-phi illusion, motion aftereffect, and rotating snakes illusion (Orger et al., 2000; Gori et al., 2014; Najafian et al., 2014). Within the context of visual motion, these studies provide behavioral evidence that mechanisms and principles similar to those of humans and other species underlie zebrafish sensory processing and associated behavior.

Characterizing aging-related changes in zebrafish behavior has important implications for our understanding of cognition and perception. First, aging-related changes in cognition are a part of the normal aging process and common in all the species. Monitoring age-dependent changes in cognition and perception is difficult to perform on the same human subject throughout life. Due to their short lifespan, behavioral assays and paradigms developed, zebrafish provides an ideal model to study cognitive and perceptual performance during aging. Second, when these behavioral studies are combined with already developed molecular and genetic tools on this aging model, we will also have a deeper understanding on the functional links between key synaptic targets, cognition, and perception during neural aging. Previous studies report significant declines in learning and memory in aged zebrafish. Typically, old zebrafish have less performance on tasks relevant with associative learning, avoidance, spatial learning and working memory (Yu et al., 2006; Arey and Murphy, 2017; Brock et al., 2017). Compared to wild-types, mutants with impaired acetylcholinesterase function had better performance in spatial learning, entrainment and increased rate of learning ( $\mathrm{Yu}$ et al., 2006; Parker et al., 2015). These findings suggest that cholinergic signaling may also play a role in age-related cognitive decline. In terms of perceptual performance, there are studies comparing larvae and adult zebrafish. However, we have limited knowledge on how perceptual performance (and thus perception and sensitivity) changes during neural aging. A challenge for the future is to characterize aging-related changes in perceptual performance and sensitivity of adult zebrafish. As mentioned above, we consider that such studies can provide comprehensive information not only on perception and behavior in general (Owsley, 2016) but also on the cellular and molecular mechanisms underlying specific aspects (e.g., motion) of perception and sensitivity.

\section{AGING-RELATED NEUROBIOLOGICAL ALTERATIONS}

Understanding the cellular mechanisms that underlie cognitive decline is important for determining sites of actions for possible interventions that could ameliorate alterations in cognitive function. Early reports indicated that age-related cognitive decline was due to significant cell (Brody, 1955; Devaney and Johnson, 1980; Henderson et al., 1980) and synapse loss (Geinisman et al., 1977; Bondareff, 1979; Curcio and Hinds, 1983; Haug and Eggers, 1991; Shi et al., 2005). However, it has become well accepted that significant cell (Haug and Eggers, 1991; Rapp and Gallagher, 1996; Rasmussen et al., 1996; Peters et al., 1998) and synapse loss does not occur in conjunction with normal aging-related declines in cognitive capacities (Poe et al., 2001; Newton et al., 2007; Shi et al., 2007). Therefore, research studies have been designed at examining markers of cellular and synaptic integrity during the aging process, such as altered neurogenesis rates (Kempermann et al., 1998, Luo et al., 2006) and the levels of key excitatory and inhibitory preand post-synaptic proteins (Newton et al., 2007; Shi et al., 2007; Adams et al., 2008), since subtle changes in cellular and synaptic functions likely underlie the aging-related declines in cognitive abilities. Moreover, examining key molecular targets that control these processes will increase our understanding of the cellular and synaptic regulation of behavior across the lifespan.

While these aging-related changes in cellular and synaptic processes could be examined in many different animal species, the zebrafish model organism is well-adapted to studying the cellular and molecular changes with aging because they have similar patterns as mammals with regards to the cellular aging process. Zebrafish on average live approximately three to five years and share a similar genome with humans (Kishi et al., 2003; Howe et al., 2013). Moreover, senescence-associated ß-galactosidase, which is a biomarker of aging, increases with advancing age in zebrafish, and this cellular alteration has been described in humans as well (Kishi et al., 2003; Arslan-Ergul et al., 2016). Finally, zebrafish have continued neurogenesis even into late adulthood (Kizil et al., 2012; Schmidt et al., 2013), they express key excitatory and inhibitory pre- and postsynaptic proteins (Karoglu et al., 2017), and classical cellular synaptic plasticity (i.e., long-term potentiation) is found in their brains (Nam et al., 2004). Recent work in the zebrafish brain has demonstrated that there are age-related declines in genes related to cellular and synaptic structure and growth (ArslanErgul and Adams, 2014), neurogenesis (Edelmann et al., 2013; Arslan-Ergul et al., 2016), and synaptic alterations (ArslanErgul et al., 2016; Karoglu et al., 2017). Interestingly, as has been shown in mammals, these changes depend on the gender of the animal (Arslan-Ergul and Adams, 2014; Karoglu et al., 2017), and the data are in good agreement with those showing sexually-dimorphic patterns published in young zebrafish brains (Ampatzis et al., 2012). Taken together, these findings indicate that the zebrafish is an appropriate model to study the effects of cellular and synaptic aging and its relationship to cognitive decline. 


\section{USE OF INTERVENTIONS TO ALTER AGING-RELATED PROCESSES}

A major goal of research related to elucidating the altered cellular and synaptic processes that underlie cognitive aging is to determine possible interventions to restore youthful cellular and synaptic function. As was mentioned previously, mutant zebrafish with lower levels of acetylcholinesterase had better performance in spatial learning, entrainment, and increased rate of learning (Yu et al., 2006; Parker et al., 2015). Therefore, these animals likely have a more youthful cellular and synaptic profile as compared to their wild-type counterparts. Currently, we are investigating this possibility and our data suggest that genetic manipulation of the cholinergic system alters the course of aging-related changes in the synaptic protein levels. We have demonstrated that at old ages as compared to their wild-type siblings, mutants have higher levels of synaptophysin, which is an indicator of presynaptic integrity, and gephyrin, a component of post-synaptic inhibitory transmission, and interestingly these changes are gender-dependent (Karoglu et al., 2018). If we can determine the cellular and synaptic profile of these mutants and how they relate to cognitive aging, it would provide potential targets for drug development to ameliorate the effects of cognitive decline.

Another potential intervention with promise is dietary restriction (DR), which is the only non-genetic intervention that reliably increases both lifespan and healthspan. Numerous studies have shown that a lifelong reduction in caloric intake from ad libitum levels increases lifespan (Roth et al., 2001; Lin et al., 2002; Colman et al., 2009). Additionally, DR increases neuronal proliferation and survival (Lee et al., 2002; Kitamura et al., 2006; Park and Lee, 2011; Park et al., 2013). We applied a short-term DR of 10 weeks and observed that this treatment did not prevent an age-related decline in cell proliferation but altered the telomere lengths of these neuronal cells (ArslanErgul et al., 2016), thereby DR exerted positive effects by subtly altering the cell cycle dynamics of these neurons. We have tested the timing and duration of short-term DR and a potential DRmimetic, rapamycin, as the positive effects of DR are thought to be modulating the mammalian target of rapamycin signaling pathway. Our data indicate that a longer duration of both DR and

\section{REFERENCES}

Adams, M. M., Shi, L., Linville, M. C., Forbes, M. E., Long, A. B., Bennett, C., et al. (2008). Caloric restriction and age affect synaptic protein levels in hippocampal CA3 and spatial learning ability. Exp. Neurol. 211, 141-149. doi: 10.1016/j.expneurol.2008.01.016

Ampatzis, K., Makantasi, P., and Dermon, C. (2012). Cell proliferation pattern in adult zebrafish forebrain is sexually dimorphic. Neuroscience 226, 367-381. doi: 10.1016/j.neuroscience.2012.09.022

Arey, R. N., and Murphy, C. T. (2017). Conserved regulators of cognitive aging: from worms to humans. Behav. Brain Res. 322, 299-310. doi: 10.1016/j.bbr.2016.06.035

Arslan-Ergul, A., and Adams, M. M. (2014). Gene expression changes in aging Zebrafish (Danio rerio) brains are sexually dimorphic. BMC Neuroscience 15:29. doi: 10.1186/1471-2202-15-29 its mimetic is more effective on aging-related changes in synaptic protein levels and transcripts, which might reflect a conserved mechanism of the beneficial effects of DR and rapamycin on life- and healthspan (Celebi-Birand et al., 2018). These studies also have the potential to provide suitable therapeutic targets around which drug development can proceed for ameliorating the devastating effects of cognitive decline.

\section{CONCLUSIONS}

The zebrafish is clearly a powerful model organism that can be used to understand the aging-related changes in both cognition and the underlying cellular and molecular processes. As previously mentioned, zebrafish exhibit characteristics that are similar to humans, as well as other mammals, including the fact that these animals age gradually, and they demonstrate aging-related changes across both cognitive and neurobiological spectrums. It clear that both genetic and nongenetic interventions can be applied to alter the course of the aging process and provide potential drug targets that could be manipulated to ameliorate age-related cognitive declines. Therefore, this model will help researchers elucidate the biological mechanisms that underlie aging-related cognitive decline.

\section{AUTHOR CONTRIBUTIONS}

All authors listed have made a substantial, direct and intellectual contribution to the work, and approved it for publication.

\section{FUNDING}

This was supported by an Installation Grant from the European Molecular Biology Organization and the Scientific and Technological Research Council of Turkey (TUBITAK 214S236 and 215S701).

\section{ACKNOWLEDGMENTS}

The authors wish to thank Elif Karoglu and Dilan Celebi-Birand for comments and discussions on the manuscript. 
Brody, H. (1955). Organization of the cerebral cortex. III. A study of aging in the human cerebral cortex. J. Comp. Neurol. 102, 511-516. doi: $10.1002 /$ cne. 901020206

Celebi-Birand, E. D., Sengul, G. F., Ardic, N. I., Kafaligonul, H., and Adams, M. M. (2018). Effects of short-term caloric restriction and rapamycin on brain aging in zebrafish (Danio rerio). Anatomy 12 (Suppl.1), 85. doi: 10.2399/ana.18.001s

Colman, R. J., Anderson, R. M., Johnson, S. C., Kastman, E. K., Kosmatka, K. J., Beasley, T. M., et al. (2009). Caloric restriction delays disease onset and mortality in rhesus monkeys. Science 325, 201-204. doi: $10.1126 /$ science. 1173635

Curcio, C. A., and Hinds, J. W. (1983). Stability of synaptic density and spine volume in dentate gyrus of aged rats. Neurobiol. Aging 4, 77-87. doi: 10.1016/0197-4580(83)90058-1

Devaney, K. O., and Johnson, H. A. (1980). Neuron loss in the aging visual cortex of man. J. Gerontol. 35, 836-841. doi: 10.1093/geronj/35.6.836

Edelmann, K., Glashauser, L., Sprungala, S., Hesl, B., Fritschie, M., Ninkovic, J., et al. (2013). Increased radial glia quiescence, decreased reactivation upon injury and unaltered neuroblast behavior underlie decreased neurogenesis in the aging zebrafish telencephalon. J. Comp. Neurol. 521, 3099-3115. doi: $10.1002 / \mathrm{cne} .23347$

Geinisman, Y., Bondareff, W., and Dodge, J. T. (1977). Partial deafferentation of neurons in the dentate gyrus of the senescent rat. Brain Res. 134, 541-545. doi: 10.1016/0006-8993(77)90828-9

Gerlai, R. (2016). Learning and memory in zebrafish (Danio rerio). Methods Cell Biol. 134, 551-586 doi: 10.1016/bs.mcb.2016.02.005

Gori, S., Agrillo, C., Dadda, M., and Bisazza, A. (2014). Do fish perceive illusory motion? Sci. Rep. 4:6443. doi: 10.1038/srep06443

Haug, H., and Eggers, R. (1991). Morphometry of the human cortex cerebri and corpus striatum during aging. Neurobiol. Aging 12, 336-338. doi: 10.1016/0197-4580(91)90013-A

Haug, M. F., Biehlmaier, O., Mueller, K. P., and Neuhauss, S. C. (2010). Visual acuity in larval zebrafish: Behavior and histology. Front. Zool. 7:8. doi: 10.1186/1742-9994-7-8

Henderson, G., Tomlinson, B. E., and Gibson, P. H. (1980). Cell counts in human cerebral cortex in normal adults throughout life using an image analysing computer. J. Neurol. Sci. 46, 113-136. doi: 10.1016/0022-510X(80)90048-9

Howe, K., Clark, M. D., Torroja, C. F., Torrance, J., Berthelot, C., Muffato, M., et al. (2013). The zebrafish reference genome sequence and its relationship to the human genome. Nature 496, 498-503. doi: 10.1038/nature12111

Kalueff, A. V., Gebhardt, M., Stewart, A. M., Cachat, J. M., Brimmer, M., Chawla, J. S., et al. (2013). Towards a comprehensive catalog of zebrafish behavior 1.0 and beyond. Zebrafish 10, 70-86. doi: 10.1089/zeb.2012.0861

Kalueff, A. V., Stewart, A. M., and Gerlai, R. (2014). Zebrafish as an emerging model for studying complex brain disorders. Trends Pharmacol. Sci. 35, 63-75. doi: 10.1016/j.tips.2013.12.002

Karoglu, E. T., Halim, D. O., Erkaya, B., Altaytas, F., Arslan-Ergul, A., Konu, O., et al. (2017). Aging alters the molecular dynamics of synapses in a sexually dimorphic pattern in zebrafish (Danio rerio). Neurobiol. Aging 54, 10-21. doi: 10.1016/j.neurobiolaging.2017.02.007

Karoglu, E. T., Tuz-Sasik, M. U., Karaduman, A., Keskus, A. G., ArslanErgul, A., Konu, O., et al. (2018). Cholinergic modulations of synaptic protein levels in male and female aged zebrafish. Anatomy 12(Suppl. 1), 25. doi: 10.2399/ana.18.001s

Kempermann, G., Kuhn, H. G., and Gage, F. G. (1998). Experience-induced neurogenesis in the senescent dentate gyrus. J. Neurosci. 18, 3206-3212. doi: 10.1523/JNEUROSCI.18-09-03206.1998

Kishi, S., Uchiyama, J., Baughman, A. M., Goto, T., Lin, M. C., and Tsai, S. B. (2003). The zebrafish as a vertebrate model of functional aging and very gradual senescence. Exp. Gerontol. 38, 777-786. doi: 10.1016/S0531-5565(03)00108-6

Kitamura, T., Mishina, M., and Sugiyama, H. (2006). Dietary restriction increases hippocampal neurogenesis by molecular mechanisms independent of NMDA receptors. Neurosci. Lett. 393, 94-96. doi: 10.1016/j.neulet.2005.08.073

Kizil, C., Kaslin, J., Kroehne, V., and Brand, M. (2012). Adult neurogenesis and brain regeneration. Dev. Neurobiol. 72, 429-461. doi: 10.1002/dneu.20918

Lee, J., Seroogy, K. B., and Mattson, M. P. (2002). Dietary restriction enhances neurotrophin expression and neurogenesis in the hippocampus of adult mice. J. Neurochem. 80, 539-547. doi: 10.1046/j.0022-3042.2001.00747.x
Lin, S. J., Kaeberlein, M., Andalis, A. A., Sturtz, L. A., Defossez, P. A., Culotta, V. C., et al. (2002). Calorie restriction extends Saccharomyces cerevisiae lifespan by increasing respiration. Nature 418, 344-348. doi: 10.1038/nature00829

Luo, J., Daniels, S. B., Lennington, J. B., Notti, R. Q., and Conover, J. C. (2006). The aging neurogenic subventricular zone. Aging Cell 5, 139-152. doi: $10.1111 / j .1474-9726.2006 .00197 . x$

Najafian, M., Alerasool, N., and Moshtaghian, J. (2014). The effect of motion aftereffect on optomotor response in larva and adult zebrafish. Neurosci. Lett. 559, 179-183. doi: 10.1016/j.neulet.2013.05.072

Nam, R. H., Kim, W., and Lee, C. J. (2004). NMDA receptor-dependent long-term potentiation in the telencephalon of the zebrafish. Neurosci. Lett. 370, 248-251. doi: 10.1016/j.neulet.2004.08.037

Neuhauss, S. C. F. (2010). "Zebrafish vision: structure and function of the zebrafish visual system," in Zebrafish, eds S. F. Perry, M. Eker, A. P. Farrell, and C. J. Brauner (London: Academic Press), 81-122.

Newton, I. G., Forbes, M. E., Linville, M. C., Pang, H., Tucker, E. W., Riddle, D. R., et al. (2007). Effects of aging and caloric restriction on dentate gyrus synapses and glutamate receptor subunits. Neurobiol. Aging 29, 1308-1318. doi: 10.1016/j.neurobiolaging.2007.03.009

Orger, M. B., and de Polavieja, G. G. (2017). Zebrafish behavior: opportunities and challenges. Аnnu. Rev. Neuro. 40, 125-147. doi: 10.1146/annurev-neuro-071714-033857

Orger, M. B., Smear, M. C., Anstis, S. M., and Baier, H. (2000). Perception of Fourier and non-Fourier motion by larval zebrafish. Nat. Neurosci. 3, 1128-1133. doi: $10.1038 / 80649$

Owsley, C. (2016). Vision and aging. Annu. Rev. Vis. Sci. 2, 255-271. doi: 10.1146/annurev-vision-111815-114550

Park, H. R., and Lee, J. (2011). Neurogenic contributions made by dietary regulation to hippocampal neurogenesis. Ann. N. Y. Acad. Sci. 1229, 23-28. doi: 10.1111/j.1749-6632.2011.06089.x

Park, J. H., Glass, Z., Sayed, K., Michurina, T. V., Lazutkin, A., Mineyeva, O., et al. (2013). Calorie restriction alleviates the age-related decrease in neural progenitor cell division in the aging brain. Eur. J. Neurosci. 37, 1987-1993. doi: $10.1111 /$ ejn.12249

Parker, M. O., Brock, A. J., Sudwarts, A., Teh, M. T., Combe, F. J., and Brennan, C. H. (2015). Developmental role of acetylcholinesterase in impulse control in zebrafish. Front. Behav. Neurosci. 9:271. doi: 10.3389/fnbeh.2015.00271

Peters, A., Morrison, J. H., Rosene, D. L., and Hyman, B. T. (1998). Feature article: are neurons lost from the primate cerebral cortex during normal aging? Cereb. Cortex 8, 295-300. doi: 10.1093/cercor/8.4.295

Poe, B. H., Linville, C., Riddle, D. R., Sonntag, W. E., and Brunso-Bechtold, J. K. (2001). Effects of age and insulin-like growth factor-1 on neuron and synapse numbers in area CA3 of hippocampus. Neuroscience 107, 231-238. doi: 10.1016/S0306-4522(01)00341-4

Rapp, P. R., and Gallagher, M. (1996). Preserved neuron number in the hippocampus of aged rats with spatial learning deficits. Proc. Natl. Acad. Sci. U.S.A. 93, 9926-9930. doi: 10.1073/pnas.93.18.9926

Rasmussen, T., Schliemann, T., Sørensen, J. C., Zimmer, J., and West, M. J. (1996). Memory impaired aged rats: no loss of principal hippocampal and subicular neurons. Neurobiol. Aging, 17, 143-147. doi: 10.1016/0197-4580(95)02032-2

Rinner, O., Rick, J. M., and Neuhauss, S. C. (2005). Contrast sensitivity, spatial and temporal tuning of the larval zebrafish optokinetic response. Invest. Ophthalmol. Vis. Sci. 46, 137-142. doi: 10.1167/iovs.04-0682

Roth, G. S., Ingram, D. K., and Lane, M. A. (2001). Caloric restriction in primates and relevance to humans. Ann. N. Y. Acad. Sci. 928, 305-315. doi: 10.1111/j.1749-6632.2001.tb05660.x

Schmidt, R., Strähle, U., and Scholpp, S. (2013). Neurogenesis in zebrafish - from embryo to adult. Neural. Dev. 8:3. doi: 10.1186/1749-8104-8-3

Shi, L., Adams, M. M., Linville, M. C., Newton, I. G., Forbes, M. E., Long, A. B., et al. (2007). Caloric restriction eliminates the aging-related decline in NMDA and AMPA receptor subunits in the rat hippocampus and induces homeostasis. Exp. Neurol. 206, 70-79. doi: 10.1016/j.expneurol.2007. 03.026

Shi, L., Linville, M. C., Tucker, E. W., Sonntag, W. E., and Brunso-Bechtold, J. K. (2005). Differential effects of aging and insulin-like growth factor1 on synapses in CA1 of rat hippocampus. Cereb. Cortex 15, 571-577. doi: 10.1093/cercor/bhh158 
Stewart, A. M., Braubach, O., Spitsbergen, J., Gerlai, R., and Kalueff, A. V. (2014). Zebrafish models for translational neuroscience research: from tank to bedside. Trends Neurosci. 37, 264-278. doi: 10.1016/j.tins.2014.02.011

Stewart, A. M., and Kalueff, A. V. (2012).The developing utility of zebrafish models for cognitive enhancers research. Curr. Neuropharmacol. 10, 263-271. doi: $10.2174 / 157015912803217323$

Tappeiner, C., Gerber, S., Enzmann, V., Balmer, J., Jazwinska, A., and Tschopp, M. (2012). Visual acuity and contrast sensitivity of adult zebrafish. Front. Zool. 9:10. doi: $10.1186 / 1742-9994-9-10$

Yu, L., Tucci, V., Kishi, S., and Zhdanova, I. V. (2006). Cognitive aging in zebrafish. PLoS ONE. 1:e14. doi: 10.1371/journal.pone.0000014
Conflict of Interest Statement: The authors declare that the research was conducted in the absence of any commercial or financial relationships that could be construed as a potential conflict of interest.

Copyright $($ () 2018 Adams and Kafaligonul. This is an open-access article distributed under the terms of the Creative Commons Attribution License (CC BY). The use, distribution or reproduction in other forums is permitted, provided the original author(s) and the copyright owner(s) are credited and that the original publication in this journal is cited, in accordance with accepted academic practice. No use, distribution or reproduction is permitted which does not comply with these terms. 